\title{
Analysis of Emotional Design in Interactive Games and Physical Interaction Mode of the Games
}

\author{
Jia-Ni Li ${ }^{1}$ and Seok-Kyoo Kim²* \\ ${ }^{1}$ Master, Student, Department of Game Design and Development, Sangmyung University, Korea. \\ ${ }^{2}$ Associate Professor, Department of Game Design and Development, Sangmyung University, Korea. \\ *Corresponding Author, ORCID: 0000-0003-4725-0288 (Seok-Kyoo Kim).
}

\begin{abstract}
This article takes the recently popular "phenomenal" new media game "Invisible Guardian" as an example. It elaborates and analyzes the emotional output of developers, user emotional experience, and emotional interaction in the game process, and then analyzes the emotional design in the new The functionality and importance of media interactive works, through the study of emotional experience issues, from the perspective of the interactive design of video games, discussed the elements of the physical interaction mode of the game that affect the emotional experience, and carried out basic experience, operating experience and environmental experience. Give examples and analysis, and summarize some interactive design suggestions that are easier to provide emotional experience for the game input and output environment.
\end{abstract}

Keywords: Interactive games, game physical interaction mode, emotional design

\section{INTRODUCTION}

When electronic game products in the market can no longer rely solely on providing pure sensory stimulation experience as a selling point, designers and players have begun to pay more and more attention to the emotional experience that games may bring. It is rare, even expensive, to devote special attention to certain emotions. As a form of entertainment, games should provide different emotional experiences. For example, movies have various types to satisfy different audiences. However, most games seen in the market can only satisfy a sense of accomplishment and power.

In the context of emotional experience, entertainment media such as movies, novels, and dramas provide one-way emotional experience transmission, either through moving plots, or through wonderful descriptions, or through highly infectious performances. . The uniqueness of the way electronic games convey emotions lies in their interactivity. The game process requires human participation. Participation lies in the player's specific operations and different choices in the process. In the "person-centered" In the new "experience economy" era, this form of entertainment respects people's free will to some extent. Therefore, emotional design is more important in new media products with real-time interactive features[1].

The design of the interactive mode of video games is essentially the design of experience. Through a certain way that not only satisfies the content of the game, but also meets the emotional needs of the player, the player can better integrate into the game. Good interactivity is an important guarantee for the sense of experience. If there is no immediate causal relationship between the operation and the feedback, there will be a gap between the player and the game, the immersion will not be produced, and the video game will lose its charm.

\section{PHYSICAL INTERACTION MODE OF THE GAMES}

The design of game physical interaction mode is to provide game players with the most basic physical meaning

The most typical interactive methods are traditional electronic game devices, such as gamepads used by home consoles, keyboards, mice, and monitors on TVs, PC platforms, and remote sensing buttons and screens on arcade machines. These devices all use von Neue Mann structure processor, including input devices for inputting data and programs, and output devices for outputting processing results. With the development of technology, game interaction modes are becoming more and more diversified. From the perspective of input methods, the emerging interaction mode called "natural interaction" has attracted more attention after reducing the cost of starting the game. Handheld, keyboard, handle, the naturalization of the operation of data gloves and somatosensory equipment increases in order. In terms of output mode, mainstream game platforms all provide image and sound output. Televisions, monitors, mobile phones, etc. have these two functions. Other more advanced outputs have also been used more and more in recent years, with enhanced auditory perception. Stereo sound, increased vibration feedback on the tactile sense, and sensory simulation that provides a three-dimensional space visually, all provide support for the improvement of game simulation and sense of 
substitution. The reliability of the interaction brought about by the reasonable design of input and output methods has become the core of immersion.

\section{INTERACTION DESIGN AND EMOTIONAL EXPERIENCE}

People who play games just sit in front of the screen and operate the device at hand. They will still be stimulated by the various activities of the character that replaces them in the virtual world. The game has a sense of substitution, and the interactive mode of the game encourages people to focus on An important influencing factor of the state, high learning costs or different interaction methods with experience habits will destroy the experience of most players; smooth interaction design makes it easier to create a complete scene and environment, and better allows users to enter State of flow, flow is not just an experience of being fully absorbed, fully engaged and enjoying it.

\section{PHYSICAL INTERACTION MODE AFFECTS THE WAY EMOTIONAL EXPERIENCE}

\section{IV.I Basic experience}

When a player first comes into contact with a game, the first information he learns is what device the game is running on, and then he knows what type of game it is through the art style and interface design. Experienced gamers can already judge this game. What is the interaction mode of Forty years ago, Yokoi Junpei invented the "cross key" to control various directional operations in the game? Although nowadays, game console hardware products have been updated for several generations, when users get the game controller, they see the "cross key". The symbol "key" can immediately understand how to use it. This symbol has been rooted in people's brains and has become part of the instinctive response. For example, when you see a game running on a PC platform using keyboard and mouse operations, people will subconsciously use the W, S, A, and D keys on the keyboard to control the movement of the game character. This is already the basic operation of electronic games. The rules, even people who have never been in the game know that using the mouse to select and click the left mouse button to select, these are all extended from the basic computer operations. The natural use of hardware devices is the foundation of the gaming experience. Game hardware itself can affect our emotions, even if we have not experienced the real content of the game, this is the "basic experience of interactive mode."

\section{IV.II Operation experience}

Interactive operation methods will affect the player's immersion. For example, in a first-person shooter game, the role played by the player often moves the mouse to aim, and then clicks the left button to correspond to the trigger in the game to shoot. The operation puts the player in the game environment well. As one of the core behaviors in shooting games, the simulation of "aiming" by moving the mouse is symbolic; the keys are similar to the real triggering for "shooting" Sex. On the other hand, the operating experience of the somatosensory game "Wii sport" is not good. It can be seen from the complex description of its rules. Compared with the traditional game that can achieve the goal by moving your fingers, this operation method combined with somatosensory has The pioneering nature of its era, but I have to admit that the complexity of its operations has far exceeded the player's mental model. Even if the player memorizes and can use these rules proficiently, he needs to spend a lot of energy to control his own body. This operation has a negative impact on the player's perception and destroys the player's gaming experience.

\section{IV.III Environmental experience}

In the game, the player will be placed in a virtual environment. The virtual environment provided by the game content will affect the player's cognition and response. The game provides goals to guide the player's operations, and the player's behavior gets feedback from the game, and the feedback provides a new game Goal, this closed loop creates a unique chain experience. The deeper this interaction is combined with the scene, the stronger the player's immersive experience. The physical interaction mode not only affects the players themselves, but more and more interactive methods allow viewers to have an interesting experience. For example, in the early years it was called "Don't Stop! The game of "Eighth Note Sauce" has become popular on social media. The rules of this game are very simple-the operation of the character to jump forward, including its simple picture, will not become a hot topic for a period of time, but its most distinctive place is The operation method is to use sound, and the pitch of the player controls the distance and height of the character's jump. In addition to the almost zero-threshold game interaction method and the freshness of sound interaction, nonparticipating players in the multiplayer scene also get interesting emotional experiences. Others are watching the game players make unusual loud or small noises. When operating a character, it even goes beyond the game player itself to get a more interesting experience. This is the entertainment effect created by the participating players in the process of interacting with the game with abnormal behavior.

\section{ANALYSIS OF EMOTIONAL DESIGN OF EXISTING INTERACTIVE GAMES}

\section{V.I Interactive game "The Invisible Guardian"}

"Invisible guardian" by the New One Studio adaptation of creative playing a game character in 2019 Nian 1 Yue $23 \mathrm{Ri}$ in Steam online gaming platform, less than a day to reach Steam hot list of the top three, favorable rate over $92 \%$, Douban scores as high as 9.6 points. Subsequently, "Invisible Guardian" launched mobile game versions for ios and Android. It is an innovative interactive video work that transforms the passive experience of live-action film and television into an interactive experience of an audio narrative publication. "The Invisible Guardian " is based on the theme 
of " spy warfare ", using live action shooting, using film and television editing and soundtrack methods to link stop motion animation and short video. The user enters the film and television environment in the game as the protagonist, and an option box will pop up along with the plot, and click to select the options provided by the system. Occasionally, simple gestures are required to unlock game clues and promote the development of the plot; finally, different ending achievements are triggered according to different choices of users. In this mobile game, movies and games are deeply integrated to form a complete interactive process.

\section{V.I.I Developer's positive emotional output}

"Invisible guardian" of the story is set in 20 century, 40 years Shanghai on behalf of the Japanese and Wang puppet government controlled by the user to the actor, "Shaw way" - a hidden underground in the perspective of the Japanese consulate, lay out Expand the storyline. As the plot develops, users need to choose different behaviors. Each choice will subtly change the trend of the next plot, and finally reach a different ending. "The Invisible Guardian" has 130 branch endings and 4 main endings. If a failed ending is reached, the player can choose to return to the storyline, restart from any stage, and continue to explore.

The game uses first-person narrative, and the plot is ups and downs. The role of the game is played by a real person and filmed in real scene. The scenes, costumes, and rituals in the screen include: the consul general of the Japanese Consulate in Shanghai whose goal is to build a strong East Asian coprosperity circle; The underground party comrades posing as unmarried couples, the military commander is placed in the undercover of the Communist Party, etc. The character identity is set in line with the background of the times, and the label dubbing is in line with the personality and identity characteristics. The game uses special sound effects and background music to render the atmosphere in key plots, highlighting emotions such as heavy sadness or tension, and giving players a sense of being there. The operation of the game is very simple. During the game, the player only needs a simple touch to complete the selection. In some scenes, the game sets up some simple gesture operations, such as swiping, long press, etc., to complete the transition of the scene or the advancement of the plot, which can not only remind the player of the importance of the plot, but also make the user more involved[2].

Developers use this combined audio-visual narrative method to stimulate the different senses of users and create a strong sense of immersion and substitution. The game interface and game operations are well-made, rigorous and meticulous, and show the developer's positive emotional output.

\section{V.I.II User rich emotional experience}

When the user opens the APP, analog protagonist " Xiao way " when the identity into the game, faced with different characters and plot, choose" their own "move to say or make. At the same time, the game provides different levels of feedback on the plot and character relationships through the user's choices. Players face various tests in "The Invisible Guardian". The life and death of the protagonist depends on the player's touch. Therefore, the game fully demonstrates the principle of empathy. Through the design of the script details, multiple ending logic and other factors, let users get rich emotional experience.

For example, in the prologue, the actor who appears to be a traitor returns to his teacher's home, and the teacher gets angry in front of other students and scolds the protagonist for "treason". In this plot, the teacher's line is "Get me where you should go, and read well ", with a close-up picture of a library card.

This plot detail laid the groundwork for the next game options. After the protagonist is kicked out of the teacher's house, the player is faced with 3 choices. Option A is " Go back to the hotel and have a good rest ", Option B is " Go to the library and read a book", and Option C is " Go to the tavern to drink and forget the troubles of today ". When the player chooses $\mathrm{A}$ or $\mathrm{C}$, the ending of "Return to Home " is triggered : Because it is not suitable for latent work, he is sent to the border area, devoted to the construction of the rear, dedicated to the grassroots, and glows. When the player chooses option B to read a book in the library, he will meet teachers and contacts in the library, accept the task arrangement in the secret room of the underground party secret contact point library, and open a new chapter. The clue of " read well " in the previous article corresponds to the library option in the following article. Such details of the story keep players immersed in the game all the time. Players need to pay attention to every line and every scene, make judgments based on different character identities, and choose relatively correct options.

In the " Extremely Evil and Inhumane " chapter of Chapter 10, one of the protagonist's tasks is to join forces with $\mathrm{Mr}$. $\mathrm{Xu}$ to shoot down the despicable Rong Yinhai. In the critical moment of being attacked by the enemy, the player needs to make a decision to " chase Rong Yinhai " or " stay to rescue $\mathrm{Mr}$. $\mathrm{Xu}$ "within 5 seconds. When the player ignores the original purpose and chooses the option of "Leave and rescue $\mathrm{Mr} . \mathrm{Xu}$ ", he will be hit by a bullet in the chaos and reach the ending of losing the other. The statement of this ending is " Before making every choice, think about it. Think of your own task" .

In such details of the story, users will experience different consequences and reach different endings through different decisions they make. The game allows users to get a rich emotional experience through real-time interaction.

\section{V.I.III Good emotional interaction}

In the plot-oriented "Invisible Guardian", in addition to the excellent objective presentation and real-time interaction process, there are more contagious and positive story themes. The background of the game is set in the country's huge turbulent situation. The protagonist, the player, has a " traitor " who appears to be a treason to Japan, and secretly 
is the dual identity of the Communist Party. The player's task is not only to please the Japanese chief and win the trust of the Japanese side, but also to steal information secretly to protect the country and the nation. The developer presents the difficult and complicated situation to the user, setting a choice between the task and the righteousness, and how to guard the ideal and original intention in the dilemma is the keynote of the deep emotional interaction between the game and the user[3].

In the sixth chapter of the game, "The Darkest Moment ", if the player fails to meet certain conditions in the previous article, he will enter the "Beautiful World " branch and join the Chongqing National Government. In this branch line of the plot, the protagonist must constantly choose flattering, false and submissive options in the development of the plot in order to please the chief executive and make money, and slander and mutilate the childhood sweethearts and classmates of the year. In the end, although the protagonist gained status and power, he died in a conspiracy and was buried in the wild, achieving a " beautiful world " ending. The ending statement is: rotten soil will only bloom the flowers of evil; souls who go astray are destined to miss the beautiful world. In this chapter, the player is pushed forward by the plot, step by step, click on the option that cannot be turned back, abandon the feelings of family and country, and finally disappear.

When the user unlocks all the key clues, improves the favorability of all the key characters to a certain level, and successfully lurks in the main line of the plot, it can finally reach the " Red Fanghua " ending. The ending statement is: After thousands of calamities, guard the return heart. In this longest plot line, the player as the protagonist must always maintain the original intention, and when appropriate, he must make the necessary sacrifices to guard the most important home country ideal in his heart.

"The righteous way in the world is the vicissitudes of life. I would like to pay tribute to the unsung heroes who once fought on the secret front with this work " is the game concept of "The Invisible Guardian". The developers use this as the theme to plan the game plot. In the interactive process of the game, the correct outlook on life, world outlook, and values will be communicated and interacted with the user, and the emotional connection with the user will make the emotional design of the game more historical and ideological .

\section{CONCLUSION}

In the new media interactive works taking "The Invisible Guardian" as an example, users experience different emotional effects under the stimulation of multiple senses such as hearing, vision, and touch, and get corresponding feedback. Thinking and attitude are potentially affected. . New media interactive works need to achieve empathy with users in emotional design, enhance the sense of substitution and immersion, and interact with users on the emotional level, so as to better express the deep-seated humanistic feelings in new media interactive works.

\section{REFERENCES}

[1] Song Binbin, Analysis of Emotional Embodiment Based on New Media Design[J], China Media Technology, 2017(5):90-91.

[2] Zhang Jiyue. Research on the Emotional Experience of Interactive Interface Design in Mobile Games $[\mathrm{J}]$. Technology and Innovation, 2019 (14): 86-87.

[3] Ni Yan. Research on the Embodiment of Emotional Elements in New Media Design [ $\mathrm{J}]$. New Media Research, 2017(18): 84-85.

[4] .Liu Zhuo. Entertainment experience and interaction design of video games[D]. Wuxi: Jiangnan University, 2008.

[5] Li Xuejing. Research on the Influence of MMORPG Player Interaction on Player Loyalty[D]. Wuhan: Huazhong University of Science and Technology, 2012. 